CLINICAL STUDY

\title{
Implication of lipocalin-2 and visfatin levels in patients with coronary heart disease
}

\author{
K M Choi ${ }^{1}$, J S Lee ${ }^{2}$, E J Kim ${ }^{3}$, S H Baik ${ }^{1}$, H S Seo ${ }^{3}$, D S Choi ${ }^{1}$, D J Oh ${ }^{3}$ and C G Park ${ }^{3}$ \\ ${ }^{1}$ Division of Endocrinology and Metabolism, Department of Internal Medicine, ${ }^{2}$ Division of Biostatistics, Graduate School of Public Health and ${ }^{3}$ Division of \\ Cardiology, Department of Internal Medicine, Korea University College of Medicine, Seoul 152-050, South Korea
}

(Correspondence should be addressed to C G Park who is now at Division of Cardiology, Department of Internal Medicine, Korea University Guro Hospital, 80 Guro-Dong, Guro-Gu, Seoul 152-050,South Korea; Email: medica7@hanmail.net)

\begin{abstract}
Objectives: Visfatin and lipocalin-2 are novel adipokines associated with insulin resistance (IR) and obesity-related metabolic disorders. We compared lipocalin-2 and visfatin concentrations between patients with coronary heart disease (CHD) and control subjects and evaluated their association with cardiovascular risk factors.

Methods: We examined serum visfatin, lipocalin-2 levels, and cardiovascular risk factors in 91 subjects (49 patients with angiographically confirmed CHD versus 42 age- and gender-matched control participants).

Results: Circulating lipocalin-2 levels were significantly higher in patients with CHD compared with the control subjects $(82.6 \pm 38.7 \mathrm{ng} / \mathrm{ml}$ versus $43.8 \pm 27.8 \mathrm{ng} / \mathrm{ml} ; P<0.001)$. However, visfatin levels were not significantly different between patients with $\mathrm{CHD}$ and control subjects. Serum lipocalin-2 levels were positively associated with weight $(r=0.26 ; P=0.036)$, fasting insulin $(r=0.36$; $P=0.003)$, and IR $(r=0.33 ; P=0.007)$, whereas these levels showed a negative correlation with high-density lipoprotein (HDL) cholesterol $(r=-0.30 ; P=0.016)$ after adjustment for gender and body mass index. However, visfatin levels were not associated with any variables of the metabolic syndrome. The multiple regression analysis showed that lipocalin-2 levels were independently associated with HDL cholesterol and IR $\left(R^{2}=0.199\right)$. Furthermore, the multiple logistic regression analysis showed that systolic blood pressure, IR, and lipocalin-2 levels were independently associated with CHD.

Conclusions: Serum lipocalin-2 levels were significantly elevated in patients with CHD and were independently associated with CHD. The present findings suggest that the measurement of serum lipocalin-2 levels may be useful for assessing CHD risk.
\end{abstract}

European Journal of Endocrinology 158 203-207

\section{Introduction}

Adipose tissue is an important endocrine organ; it secretes several hormones and cytokines that are involved in the metabolic syndrome (1). Adipokines, signaling proteins secreted by adipose tissue, have an important regulatory function throughout the body; they include leptin, tumor necrosis factor- $\alpha$ (TNF- $\alpha$ ), interleukin-6 (IL-6), resistin, and adiponectin. Lower serum adiponectin levels have been reported in patients with coronary heart disease (CHD) $(2,3)$ and have been shown to be a risk factor for cardiovascular events (4). In addition, it has recently been suggested that IL-6, TNF- $\alpha$, leptin, and adiponectin may not only be just markers of inflammation and cardiovascular (CV) risk but are also likely to play a pathogenic role in atheromatous plaque $(5,6)$.

Recently, lipocalin-2, also known as neutrophil gelatinase-associated lipocalin and $24 \mathrm{p} 3$, was reported to be associated with obesity and insulin resistance (IR) in both mice and humans (7). In $d b / d b$ obese diabetic mice, circulating lipocalin-2 concentrations were increased as well as the expression of lipocalin-2 in adipose and liver tissue compared with normal mice. In addition, serum lipocalin-2 levels were positively correlated with body mass index (BMI), hypertriglyceridemia, hyperglycemia, and IR, but negatively correlated with high-density lipoprotein (HDL) cholesterol in humans (7). Treatment with rosiglitazone markedly decreased lipocalin-2 expression in mice and circulating levels in both mice and humans (7). Furthermore, there was a recent report that expression of lipocalin-2 is increased in atherosclerotic plaques and myocardial infarction (8). Recently, Aigner et al. reported that lipocalin-2 regulates the inflammatory response during ischemia and reperfusion of the transplanted heart (9). Lipocalin families share a common tertiary structure formed by segments termed lipocalin folds (10). 
One of the human lipocalins is retinol-binding protein 4, recently identified as an adipokine that may link obesity and $\operatorname{IR}(11,12)$.

Visfatin is a novel adipokine that is preferentially produced in visceral adipose tissue; both its expression and plasma concentration increase with increasing levels of obesity (13). Visfatin treatment has exhibited insulin-mimetic activity resulting in a glucose-lowering effect (13), and increasing concentrations of visfatin were independently and significantly associated with type 2 diabetes (14). However, another study reported that there was no correlation between visfatin levels and various parameters of IR during euglycemic-hyperinsulinemic clamp experiments (15). Therefore, the previous studies evaluating the association between visfatin levels and diabetes, obesity, or dyslipidemia have yielded inconsistent results (16).

Although obesity and its associated conditions including the metabolic syndrome are closely associated with the development of CHD, there was no prior study reporting on an association between CHD and these novel adipokines. Therefore, the aim of the present study was to evaluate circulating lipocalin-2 and visfatin levels in patients with CHD and compare these levels with control subjects, and to determine their association with other cardiovascular risk factors for atherosclerosis.

\section{Subjects and methods}

\section{Study subjects}

The study group included 49 patients with an established diagnosis of CHD after coronary angiography (CAG) performed in cardiovascular centers of Korea University Guro Hospital. All clinical and angiographic data were stored and analyzed using CAG database system of Korea University Guro Hospital. Patients were divided into three groups: acute myocardial infarction (AMI), unstable angina pectoris (UAP), or stable angina pectoris (SAP). AMI was diagnosed on the basis of clinical symptoms, electro cardiogram (ECG) evidence of a $>0.1 \mathrm{mV}$ ST segment elevation in at least two leads (in cases of ST elevation myocardial infarction), and a greater than twofold increase in the level of serum creatine kinase-MB isoform concentration from the upper limit of the normal range. CAG confirmed the occlusion of a coronary artery with a TIMI grade flow of $<3$. UAP was diagnosed on the basis of clinical symptoms (class IB, IIB, and IIIB in the Braunwald classification), a CAG finding of more than $70 \%$ stenosis in $\geq 1$ coronary arteries, and no significant elevation in the level of serum creatine kinase-MB concentration. SAP was diagnosed based on typical chest pain during an exercise test and the CAG findings of more than 70\% stenosis in $\geq 1$ coronary arteries. The control group consisted of 42 healthy participants with normal routine biochemistry evaluations, normal ECG, and matched by age and gender to the patients with CHD. The study exclusion criteria adopted were: a previous diagnosis or treatment of diabetes, major trauma or surgery, active infectious disease, malignant disease, and liver or renal dysfunction. Informed consent was obtained from all subjects before they participated in the study, which was approved by the ethical committee at our institution.

\section{Measurements of risk variables}

The BMI was calculated as weight $/$ height $^{2}\left(\mathrm{~kg} / \mathrm{m}^{2}\right)$. Waist circumference was measured from the narrowest point between the lower borders of the rib cage and the iliac crest. Blood samples were obtained at least 3 months after an acute event. All blood samples were obtained in the morning after a 12 -h overnight fast and were immediately stored at $-80^{\circ} \mathrm{C}$ for subsequent assay. Serum triglycerides and HDL cholesterol were determined enzymatically using a chemistry analyzer (Hitachi 747). A glucose oxidase method was employed to measure plasma glucose, and a human insulinspecific RIA kit (Linco Research Inc., St Charles, MO, USA) was used to measure insulin levels. This kit had a reactivity of $<0.2 \%$ with human proinsulin. IR was calculated by the homeostasis model assessment (HOMA) (17). Serum visfatin levels were measured using a Visfatin Human ELISA kit (Phoenix pharmaceuticals, Belmont, CA, USA), with an intra-CV of $4.4 \%$. Serum lipocalin-2 levels were determined using a Human Lipocalin-2 Quantikine ELISA kit (R\&D Systems, Minneapolis, MN, USA), with an intra-CV of $1.0 \%$.

\section{Statistical analysis}

Data are expressed as means \pm s.D. or median and interquartile range. Non-normally distributed variables were analyzed using log-transformed values. We used the Kolmogorov-Smirnov test to evaluate variables for normality. Differences between groups were tested using the unpaired Student's $t$-test or the Mann-Whitney $U$ test. Categorical variables were compared by the $\chi^{2}$-test. The spearman rank correlation test was performed to determine the relationships between lipocalin-2, visfatin levels, and other cardiovascular risk variables. Multiple regression analysis was performed with lipocalin-2 concentrations as a dependent variable. Age, gender, smoking status, weight, BMI, waist circumference, systolic blood pressure, diastolic blood pressure, HDL cholesterol, triglyceride, fasting glucose, and HOMA-IR levels were employed as independent variables. The stepwise method was used for significant variable selection. Multivariate logistic regression analysis using the existence of $\mathrm{CHD}$ as a dependent variable was conducted to determine the relative contributions made by each variable to the outcome variable. Variables used in multiple regression analysis plus visfatin and lipocalin-2 levels were used as independent 
variables. Significant independent variables were chosen using the backward: conditional variable selection method. Values of $P<0.05$ were considered significant. Data were analyzed using SPSS for Windows (version 10.0; SPSS Inc., Chicago, IL, USA).

\section{Results}

Clinical and biochemical characteristics of the study subjects are presented in Table 1. Patients with CHD exhibited greater body weight, BMI, fasting glucose, fasting insulin levels, and HOMA-IR compared with the control subjects. However, age, gender, blood pressure, lipid profile, and smoking status were not different in comparisons between patients with $\mathrm{CHD}$ and control participants.

Circulating lipocalin-2 levels were significantly higher in patients with $\mathrm{CHD}$ compared with the control subjects $(82.6 \pm 38.7 \mathrm{ng} / \mathrm{ml}$ versus $43.8 \pm 27.8 \mathrm{ng} / \mathrm{ml} ; P<0.001$; Fig. 1). However, visfatin levels were not different between

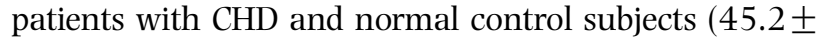
$20.9 \mathrm{ng} / \mathrm{ml}$ versus $44.4 \pm 21.0 \mathrm{ng} / \mathrm{ml} ; P=0.761)$. Interestingly, even when patients with $\mathrm{CHD}$ were compared with age-, gender-, and BMI-matched control subjects $(n=26$, BMI 25.6 \pm 2.1$)$, serum lipocalin-2 levels were still significantly higher in patients with $\mathrm{CHD}(82.6 \pm$ $38.7 \mathrm{ng} / \mathrm{ml}$ versus $44.6 \pm 28.9 \mathrm{ng} / \mathrm{ml} ; P<0.001)$. Patients with AMI or UAP $(n=11)$ showed a tendency for increasing serum lipocalin-2 levels compared with the patients with SAP $(n=38)$, although this finding was not statistically significant $(91.7 \pm 32.7$ vs $79.9 \pm$ $40.3 \mathrm{ng} / \mathrm{ml} ; P=0.326$ ). Lipocalin-2 concentrations from

Table 1 Clinical and laboratory characteristics of study subjects.

\begin{tabular}{lccc}
\hline & $\begin{array}{c}\text { Control } \\
(n=42)\end{array}$ & $\begin{array}{c}\text { CHD } \\
(n=49)\end{array}$ & $\boldsymbol{P}$ \\
Variables & $25 / 17$ & $30 / 19$ & 0.869 \\
\hline Sex (M/F) & $61.1 \pm 7.8$ & $60.6 \pm 9.4$ & 0.793 \\
Age (years) & $161.3 \pm 9.4$ & $161.7 \pm 7.6$ & 0.801 \\
Height (cm) & $63.2 \pm 8.9$ & $68.2 \pm 8.3$ & 0.007 \\
Weight (kg) & $24.4 \pm 2.4$ & $26.1 \pm 2.6$ & 0.002 \\
$\begin{array}{c}\text { Body mass index } \\
\quad\left(\mathrm{kg} / \mathrm{m}^{2}\right)\end{array}$ & & & \\
Waist circumference & $85.7 \pm 6.6$ & $88.0 \pm 6.5$ & 0.135 \\
$\quad(\mathrm{~cm})$ & & & \\
SBP (mmHg) & $130.6 \pm 12.9$ & $128.0 \pm 11.2$ & 0.320 \\
DBP (mmHg) & $79.9 \pm 9.8$ & $78.9 \pm 8.4$ & 0.634 \\
HDL cholesterol & $1.3(1.2,1.5)$ & $1.3(1.1,1.6)$ & 0.569 \\
$\quad(\mathrm{mmol} / \mathrm{l})$ & & & \\
Triglyceride (mmol/l) & $1.1(0.8,1.7)$ & $1.2(1.0,1.7)$ & 0.393 \\
Fasting glucose & $5.1 \pm 1.0$ & $5.7 \pm 1.0$ & 0.003 \\
$\quad(\mathrm{mmol} / \mathrm{l})$ & & & \\
Fasting insulin & $9.1(6.7,11.3)$ & $13.5(11.3,18.8)$ & $<0.001$ \\
$\quad(\mu \mathrm{U} / \mathrm{ml})$ & & & \\
HOMA-IR & $1.9(1.6,2.5)$ & $3.4(2.8,4.6)$ & $<0.001$ \\
Smoking, $n(\%)$ & $12(28.6 \%)$ & $15(30.6 \%)$ & 0.832 \\
\hline
\end{tabular}

Data presented as means \pm S.D. or median (inter-quartile range). SBP, systolic blood pressure; DBP, diastolic blood pressure; HDL, high-density lipoprotein; and HOMA-IR, homeostasis model assessment insulin resistance.

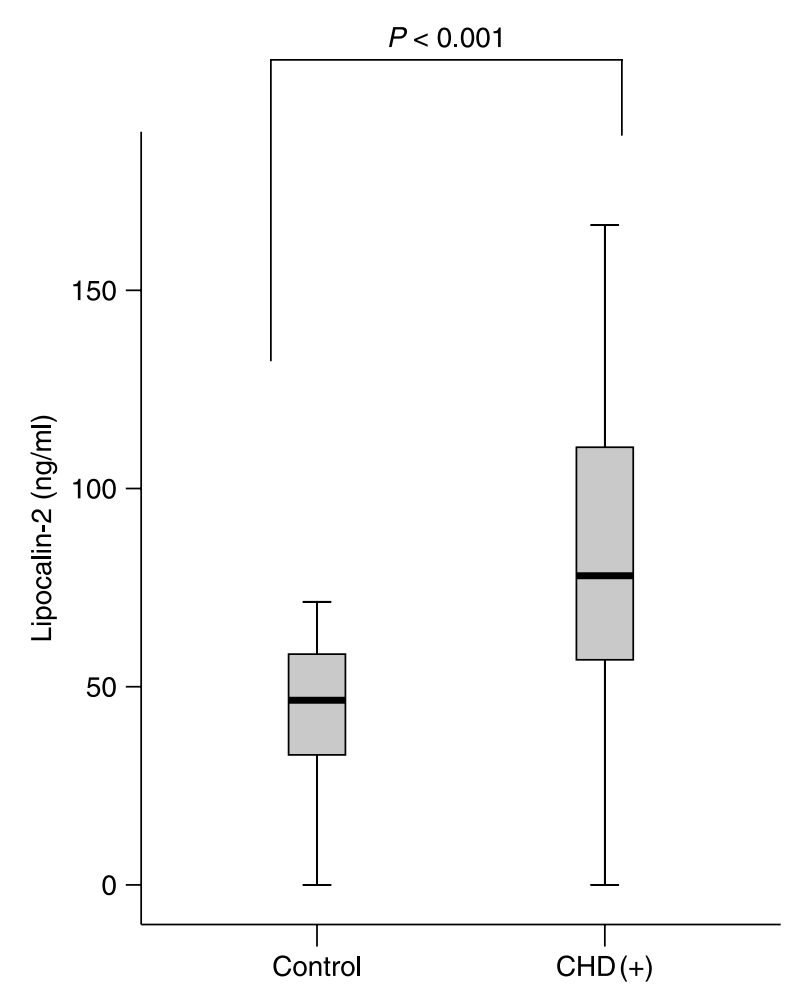

Figure 1 Serum lipocalin-2 concentrations in patients with coronary heart diseases and in control subjects. The box plot displays the 25th, median and 75th percentiles, and the minimum and maximum levels as horizontal lines outside the box.

men also exhibited higher levels compared with those from women $(70.4 \pm 40.1$ vs $55.9 \pm 36.3 \mathrm{ng} / \mathrm{ml}$; $P=0.091)$.

In Table 2, results of partial correlation analysis adjusted for gender and BMI between serum lipocalin-2 and other cardiovascular risk factors are shown. Serum lipocalin-2 levels were positively associated with body weight $(r=0.26 ; P=0.036)$, fasting insulin $(r=0.36$; $P=0.003)$, and IR $(r=0.33 ; P=0.007)$, whereas

Table 2 Partial correlation analysis adjusted for gender and body mass index.

\begin{tabular}{lrc}
\hline & \multicolumn{2}{c}{ Lipocalin-2 } \\
\cline { 2 - 3 } & $r$ & $P$ \\
\cline { 2 - 3 } Age & -0.06 & 0.645 \\
Height & 0.21 & 0.086 \\
Weight & 0.26 & 0.036 \\
Waist circumference & 0.18 & 0.161 \\
Systolic blood pressure & -0.24 & 0.057 \\
Diastolic blood pressure & -0.16 & 0.214 \\
HDL cholesterol & -0.30 & 0.016 \\
Triglyceride & 0.07 & 0.578 \\
Fasting glucose & 0.00 & 0.975 \\
Fasting insulin & 0.36 & 0.003 \\
HOMA-IR & 0.33 & 0.007
\end{tabular}

HDL, high-density lipoprotein and HOMA-IR, homeostasis model assessment insulin resistance. 
Table 3 Multiple regression analysis with lipocalin-2 concentrations as a dependent variable.

\begin{tabular}{|c|c|c|c|c|}
\hline & \multicolumn{2}{|c|}{$\begin{array}{l}\text { Unstandardized } \\
\text { coefficients }\end{array}$} & \multicolumn{2}{|c|}{$\begin{array}{l}\text { Standardized } \\
\text { coefficients }\end{array}$} \\
\hline & $\beta$ & S.E.M. & $\beta$ & $P$ \\
\hline Constant & 5.025 & 0.737 & & $<0.001$ \\
\hline $\begin{array}{l}\text { HDL } \\
\text { cholesterol }\end{array}$ & -0.036 & 0.011 & -0.361 & 0.002 \\
\hline HOMA-IR & 0.226 & 0.113 & 0.225 & 0.050 \\
\hline
\end{tabular}

$R^{2}=0.199 . \mathrm{HDL}$, high-density lipoprotein and HOMA-IR, homeostasis model assessment insulin resistance.

they had a negative correlation with HDL cholesterol $(r=-0.30 ; P=0.016)$. However, circulating visfatin levels were not significantly associated with variables such as obesity, blood pressure, lipid profile, and IR. The multiple regression analysis showed that lipocalin-2 levels were independently associated with HDL cholesterol and HOMA-IR $\left(R^{2}=0.199\right.$; Table 3$)$. Multiple logistic regression analysis was performed using the presence of CHD as a dependent variable. CHD was associated with systolic blood pressure, HOMA-IR, and lipocalin-2 levels independently from other cardiovascular risk factors (Table 4).

\section{Discussion}

The present study has shown for the first time that serum lipocalin-2 levels were significantly higher in patients with $\mathrm{CHD}$ than in age- and gender-matched control subjects. Furthermore, serum lipocalin-2 levels were independently associated with HDL cholesterol and IR. However, circulating visfatin levels were not significantly related to cardiovascular risk variables such as obesity, blood pressure, lipid profile, and IR and were not different in comparisons between patients with CHD and control subjects.

Chronic low-grade inflammation is now recognized to be a key mediator in the development of CHD (18). Epidemiologic studies have shown a consistent independent association between high-sensitivity C-reactive protein (hs-CRP) elevations and coronary risk (19). Wang et al. reported a strong positive association between lipocalin-2 concentrations and hs-CRP, independent of age, sex, and adiposity $(P=0.007)$ and suggested that lipocalin-2 can be considered a marker of obesity-related low-grade inflammation (7). Recently, Hemdahl et al. have shown that mice developing myocardial infarction exhibited increased lipocalin-2 expression in coronary atherosclerotic plaques (8).

Wang et al. exhibited that adipose tissue and liver are two principal sources contributing to increased lipocalin-2 levels in obese states. They also reported a significant positive correlation between lipocalin-2 levels and several variables associated with obesityrelated metabolic disorders, including adverse lipid profiles, hyperinsulinemia, hyperglycemia, and IR (7). In the present study, we found that circulating lipocalin-2 levels were positively associated with body weight, fasting insulin, and HOMA-IR, whereas they were negatively associated with HDL cholesterol, consistent with the prior results. However, we could not find any relationship between BMI, waist circumference, triglyceride, fasting glucose, and lipocalin-2 levels. Of note is that our study included subjects with relatively narrow range of BMI compared with the prior study subjects (7).

Visfatin is a recently identified adipokine that is preferentially secreted by visceral adipocytes. Visfatin binds to and activates insulin receptors, and decreases IR (13). Chen et al. reported that visfatin concentration was elevated in type 2 diabetes mellitus even after statistical adjustment for known biomarkers (14). They also reported that plasma visfatin was associated with age, waist-to-hip ratio (WHR), fasting insulin, adiponectin levels, and HOMA-IR in a simple regression analysis, whereas in a multiple regression analysis, only WHR remained positively associated with plasma visfatin levels. However, we could not confirm these relationships in our study subjects. Our results are compatible with the recent observations based on investigation of a community-based sample; this suggested that circulating visfatin may not be a useful clinical biomarker for metabolic traits (16). Furthermore, there was no difference identified in circulating visfatin levels between subjects with CHD and control subjects in the present study, although a recent study reported increased expression of visfatin in macrophages of human unstable carotid and coronary atherosclerosis (20).

Because of the limitations of cross-sectional study in the present study, no causal relationship could be defined. It is not clear whether increased lipocalin-2 in patients with CHD is a causative factor or simply a bystander in the pathogenesis of atherosclerosis. In addition, normal ECG

Table 4 Multivariate logistic regression analysis with coronary heart disease as a dependent variable.

\begin{tabular}{lcccccc}
\hline & $\boldsymbol{B}$ & S.E.M. & Wald & $\boldsymbol{P}$ & Exp $(B)$ & $\mathbf{9 5 \%} \mathbf{C l}$ \\
\hline SBP & 0.091 & 0.059 & 2.364 & 0.124 & 1.095 & 0.975 \\
HOMA-IR & 5.293 & 1.965 & 7.253 & 0.007 & 199 & 1.229 \\
Lipocalin-2 & 0.045 & 0.023 & 3.835 & 0.050 & 1.046 & 9374 \\
Constant & -28.635 & 11.737 & 5.952 & 0.015 & 0.000 & 1.000 \\
\end{tabular}

SBP, systolic blood pressure and HOMA-IR, homeostasis model assessment insulin resistance. 
in the control group does not completely exclude the presence of CHD particularly in elderly subjects.

In conclusion, circulating concentrations of lipocalin2 were significantly higher in patients with CHD, and were independently associated with CHD. However, visfatin levels were not associated with cardiovascular risk factors for atherosclerosis. Further, prospective study with a large number of patients is needed to determine the predictive value of serum lipocalin-2 as a biomarker for metabolic and cardiovascular disease.

\section{Acknowledgements}

This work was supported by the Korea Science and Engineering Foundation (KOSEF) grant funded by the Korea government (no. R01-2007-000-20546-0) and Seoul R\&BD Program, Republic of Korea (A 10526).

\section{References}

1 Kershaw EE \& Flier JS. Adipose tissue as an endocrine organ. Journal of Clinical Endocrinology and Metabolism 200489 2548-2556.

2 Kumada M, Kihara S, Sumitsuji S, Kawamoto T, Matsumoto S, Ouchi N, Arita Y, Okamoto Y, Shimomura I, Hiraoka H, Nakamura T, Funahashi T \& Matsuzawa Y. Association of hypoadiponectinemia with coronary artery disease in men. Arteriosclerosis, Thrombosis, and Vascular Biology 200323 85-89.

3 Pischon T, Girman CJ, Hotamisligil GS, Rifai N, Hu FB \& Rimm EB. Plasma adiponectin levels and risk of myocardial infarction in men. Journal of the American Medical Association 2004291 1730-1737.

4 Rothenbacher D, Brenner H, Marz W \& Koenig W. Adiponectin, risk of coronary heart disease and correlations with cardiovascular risk markers. European Heart Journal 200526 1640-1646.

5 Karaduman M, Sengul A, Oktenli C, Pekel A, Yesilova Z, Musabak U, Sansioglu SY, Gunay C, Baysan O, Kocar IH, Tatar H \& Ozata M. Tissue levels of adiponectin, tumour necrosis factor-alpha, soluble intercellular adhesion molecule-1 and heart-type fatty acid-binding protein in human coronary atherosclerotic plaques. Clinical Endocrinology 200664 196-202.

6 Karaduman M, Oktenli C, Musabak U, Sengul A, Yesilova Z, Cingoz F, Olgun A, Sanisoglu SY, Baysan O, Yildiz O, Taslipinar A, Tatar H, Kutlu M \& Ozata M. Leptin, soluble interleukin-6 receptor, C-reactive protein and soluble vascular cell adhesion molecule-1 levels in human coronary atherosclerotic plaque. Clinical and Experimental Immunology $2006 \mathbf{1 4 3} 452-457$.

7 Wang Y, Lam KS, Kraegen EW, Sweeney G, Zhang J, Tso AW, Chow WS, Wat NM, Xu JY, Hoo RL \& Xu A. Lipocalin-2 is an inflammatory marker closely associated with obesity, insulin resistance, and hyperglycemia in humans. Clinical Chemistry 200753 34-41.

8 Hemdahl AL, Gabrielsen A, Zhu C, Eriksson P, Hedin U, Kastrup J, Thoren P \& Hanssor GK. Expression of neutrophil gelatinaseassociated lipocalin in atherosclerosis and myocardial infarction. Arteriosclerosis, Thrombosis, and Vascular Biology 200626 136-142.
9 Aigner F, Maier HT, Schwelberger HG, Wallnofer EA, Amberger A, Obrist P, Berger T, Mak TW, Maglione M, Margreiter R, Schneeberger S \& Troppmair J. Lipocalin-2 regulates the inflammatory response during ischemia and reperfusion of the transplanted heart. American Journal of Transplantation $2007 \mathbf{7}$ 779-788.

10 Borregaard N \& Cowland JB. Neutrophil gelatinase-associated lipocalin, a siderophore-binding eukaryotic protein. Biometals 200619 211-215.

11 Yang Q, Graham TE, Mody N, Preitner F, Peroni OD,, Zabolotny JM, Kotani K, Quadro L \& Kahn BB. Serum retinol binding protein 4 contributes to insulin resistance in obesity and type 2 diabetes. Nature 2005436 356-362.

12 Graham TE, Yang Q, Bluher M, Hammarstedt A, Ciaraldi TP, Henry RR, Wason CJ, Oberbach A, Jansson PA, Smith U \& Kahn BB. Retinol-binding protein 4 and insulin resistance in lean, obese, and diabetic subjects. New England Journal of Medicine 2006 $3542552-2563$.

13 Fukuhara A, Matsuda M, Nishizawa M, Segawa K, Tanaka M, Kishimoto K, Matsuki Y, Murakami M, Ichisaka T, Murakami H, Watanabe E, Takagi T, Akiyoshi M, Ohtsubo T, Kihara S, Yamashita $S$, Makishima $M$, Funahashi T, Yamanaka $S$, Hiramatsu R, Matsuzawa Y \& Shimomura I. Visfatin: a protein secreted by visceral fat that mimics the effects of insulin. Science $2005307426-430$.

14 Chen MP, Chung FM, Chang DM, Tsai JC, Huang HF, Shin SJ \& Lee YJ. Elevated plasma level of visfatin/pre-B cell colony-enhancing factor in patients with type 2 diabetes mellitus. Journal of Clinical Endocrinology and Metabolism 200691 295-299.

15 Berndt J, Kloting N, Kralisch S, Kovacs P, Fasshauer M, Schon MR, Stumvou M \& Bluher M. Plasma visfatin concentrations and fat depot-specific mRNA expression in humans. Diabetes $2005 \mathbf{5 4}$ 2911-2916.

16 Ingelsson E, Larson MG, Fox CS, Yin X, Wang TJ, Lipinska I, Pou KM, Hoffmann U, Benjamin EJ, Keaney JF, Jr \& Vasan RS. Clinical correlates of circulating Visfatin levels in a communitybased sample. Diabetes Care $2007301278-1280$.

17 Matthews DR, Hosker JP, Rudenski AS, Naylor BA, Treacher DF \& Turner RC. Homeostasis model assessment: insulin resistance and beta-cell function from fasting plasma glucose and insulin concentrations in man. Diabetologia 198528 412-419.

18 Tousoulis D, Charakida M \& Stefanadis C. Endothelial function and inflammation in coronary artery disease. Heart 200692 441-444.

19 Gotto AM Jr. Role of C-reactive protein in coronary risk reduction: focus on primary prevention. American Journal of Cardiology 2007 $99718-725$.

20 Dahl TB, Yndestad A, Skjelland M, Oie E, Dahl A, Michelsen A, Damas JK, Tunheim SH, Ueland T, Smith C, Bendz B, Tonstad S, Gullestad L, Froland SS, Krohg-Sorensen K, Russell D, Aukrust P \& Halvorsen B. Increased expression of visfatin in macrophages of human unstable carotid and coronary atherosclerosis: possible role in inflammation and plaque destabilization. Circulation 2007 $115972-980$.

Received 28 October 2007

Accepted 22 November 2007 